\title{
Design and Implementation of Cost Efficient SCADA System for Industrial Automation
}

\author{
Sudip Phuyal ${ }^{\mathrm{a}, *}$, Diwakar Bista ${ }^{\mathrm{a}, *}$, Jan Izykowski ${ }^{\mathrm{b}, *}$, Rabindra Bista ${ }^{\mathrm{c}, *}$ \\ ${ }^{a}$ Department of Electrical and Electronics Engineering, Kathmandu University Dhulikhel 45200, Nepal \\ ${ }^{b}$ Faculty of Electrical Engineering, Wroclaw University of Science and Technology, Wroclaw 50-370, Poland \\ ${ }^{c}$ Department of Computer Science and Engineering, Kathmandu University, Dhulikhel 45200, Nepal
}

Received: 27 December 2019; Accepted: 27 January 2020; Published: 08 April 2020

\begin{abstract}
Supervisory Control and Data Acquisition system facilitates the monitoring and control of industrial process from the designated control station. The cost investment for implementing the SCADA system is expensive and is not in approach of small and medium scale industries these days. Developing the customized SCADA system helps to reduce the cost in software components and becomes more close approach to the small and medium scale industries. In this research we presented a SCADA software developed in C\# environment and successfully tested in industrial process monitoring and control. The developed SCADA software is capable of remotely supervise, control and data monitoring facility and is also capable of data logging in the IoT server. This approach has been found to be efficient in both aspects, technically and economically.

Index Terms: Low Cost SCADA, Remote Monitoring, Industrial Automation, Industry 4.0, Cyber Physical System, Internet of Things.

(C) 2020 Published by MECS Publisher. Selection and/or peer review under responsibility of the Research Association of Mode rn Education and Computer Science
\end{abstract}

* Corresponding author.

E-mail address:sudip.phuyal@student.ku.edu.np(S.P.), diwakarbista@ku.edu.np (D.B.), jan.izykowski@pwr.edu.pl (J.I.), rbista@ku.edu.np (R.B.) 


\section{Introduction}

Supervisory Control and Data Acquisition (SCADA) is an integration of software and hardware components facilitating supervision and control of industrial or any processes remotely or locally [1]. The system to be monitored and controlled involves the real time operation and data logging, process handling and supervisory control access. The SCADA unit facilitates the observation and control of all the pant process easily and more conveniently. In the current scenario, the SCADA system has been used more widely and its scope is increasing day by day, but due to the higher costs, the smaller and medium scale industries are in the shade of using SCADA system in monitoring of their plant processes. Furthermore, in this rapidly developing market, a successful digitization of production can play a vital role in stepping forward towards the modern age [2, 3]. For the European manufacturing industries, the digitization and the modern manufacturing have been supported by Industry 4.0 [4], the factory of the future [5] and ProcessIT.EU roadmap on process automation [6]. By reducing the cost investment in the SCADA software and hardware system, it would be more accessible and its use and area of implementation would also get increased, and the cost can be reduced by using open-source software tools to develop own SCADA system and also the customization of the developed software is also possible depending on the requirements.

In this article, we propose the cost-efficient SCADA system developed in C\# environment. The performance has been evaluated under testing environment in an industry. This approach of SCADA helps to eliminate the investment in purchasing the licensed SCADA software tools, and also gives the efficient performance which is comparable to the paid software. The first section of this paper introduced about the contribution of this paper in development of low-cost SCADA system along with its associated terms, its importance in power and process monitoring and control system, the second section describes related works in this field and the latest innovations in development of low cost SCADA system, third section explains the proposed system development methodology, fourth section explains the experimental setup and the hardware implementation and fifth section concludes the work with necessary discussions.

\begin{tabular}{|ll}
\hline \multicolumn{2}{|l}{ Nomenclature } \\
API & Application Program Interface \\
EU & European Union \\
IO & Input and Output \\
IoT & Internet of Things \\
IP & Internet Protocol \\
IR & Infrared \\
IT & Information Technology \\
MCCB & Moulded Case Circuit Breaker Metropolitan \\
MUN & Area Network \\
PC & Personal Computer \\
PLC & Programmable Logic Controller \\
PV & Photo-Voltaic \\
RS485 & Recommended Standard-485 \\
RTU & Remote Terminal Unit \\
RTU & Remote Terminal Unit \\
SCADA & Supervisory Control and Data Acquisition \\
VFD & Variable Frequency Drive \\
WAN & Wide Area Network \\
XOR & Exclusive OR \\
\hline
\end{tabular}




\section{Related Works}

Over the globe the major complexities with the SCADA system for the small and medium scale industry is found to be the higher installation cost and the compatibility of the system. Various research is being conducted for optimization of the investment cost in the SCADA system without compromising the most essential and crucial needs of the system. The SCADA system is required to be powerful with the features like security, visualization, controlling of process parameters, automatic handling of the process, warning system etc.

The industrial automation system is the interdisciplinary system which is inter-related with electrical, electronics, communication, mechanical and chemical engineering. Aghenta et. al. [1] proposed low cost IoT based web SCADA system using thinger.IO and ESP32 thing in which they developed microcontroller based energy monitoring and controlling system in web interface and Mercurio et.al. also implemented web-based IEC standard SCADA system to reduce the investment cost without compromising the performance of the system [7]. Alihussein [8] has tested the performance of SCADA system in different communication medium such as cellular connection, ethernet, RS 485 and concluded RS 485 is easier for hardware interconnection and communication in industrial environment. Ahmed [9] also presented the research regarding the implementation of web-based SCADA system focusing in real time operation in dynamic web interface. Carlsson [10] presented his work regarding the implementation of IoT in industrial application and suggested the real time monitoring of industrial process through the use of IoT has been found to be more efficient for remotely accessing the industrial process. The SCADA system which are sensitive and need continuous monitoring and observation needs to be implemented in static applications. In [11] the Arduino Uno microcontroller is used along with Zigbee module to maintain a wireless sensor network containing flow sensor, temperature sensor and ultrasonic sensor. The actuators are used to maintain heat, control valve and pump water. It was able to provide a data through a computer developing an economical system. In [12], recent technology cloud computing is used to connect the network of sensor in a single unit. They used STM32F407 microprocessors with Bosch BMI160 inertial measurement units connected by means of ethernet. With the growth of wireless technology, the microcontroller-based SCADA system are widely studied and applied. MODBUS and ATmega 2560 were used in [13] Vijeo Citect v7.2 (SCADA software) is used to operate and monitor the SCADA components. The ATMega 2560 micro-controller was used as RTU for sensor data collection After that it forms a response frame to the corresponding MODBUS query All the sequences were performed in orderly manner at definite interval of time.

Open source SCADA was used in [14] and can be used as usual SCADA function. The operations are performed under Linux operating system. Open source code could be used in SCADA system which help to execute the operation in real time. The problems of real time control system could be solved using Open source code. Paper [15] also used open source SCADA system for process monitoring which used open source in different form with machine communication using OPC unified architecture. IoT based SCADA system was developed in paper [16]. The paper discusses about the PV monitoring system using open source low cost SCADA system. They have used Arduino Uno as a remote terminal unit for the data acquisition through the raspberry Pi connected to Metropolitan Area Network (MUN). Going through different research works, we can get overall procedure of the SCADA system with different components, communication medium and visualization system. Most of them are low cost and built mostly from open source. They have developed different experimental set-up to verify the SCADA system with different methodology dedicating IOT based SCADA system as future.

As seen from various studies, the emerging research is being conducted in the field of optimizing the cost investment of the supervisory control and data acquisition system for making more reliable and efficient 
industrial automation system. Based on some related works, we developed advanced control system based on windows 32-bit application developed in C\# environment and the Schneider M221 series PLC for the hardware interface for the developed system. The developed hardware and software have been implemented in an industry to test the performance and compare it with the paid versions of the same system.

\section{Description of Designed System}

The main user interface of the SCADA system is developed in C\# environment with the feature of controlling and monitoring the system through internet/cloud server. The communication of SCADA system to the IoT cloud is through the internet protocol and the connection has been secured through end to end encryption. The IoT server data can be accessed through the internet from any mobile devices after the successful login with pre-shared API key and password. The operation and control actions can be performed from multiple devices and also be monitored in multiple screens. The real-time operation data is managed to logged into the cloud server and the detailed report can be generated as per requirement of the user.

The remote terminal unit is set-up with PLC through Modbus communication through RS-485 communication channel. The PLC and the SCADA computer is installed in the same local network through ethernet cable to the router and router is connected to WAN for operation of the system through IoT devices. Fig. 1 shows the overall architecture of the proposed system. The power is supplied to the machines through Variable Frequency Drive (VFD) and the control signal is also sent through the VFD to run the machines in the desired manner. The RS-485 link establishes the communication between PLC and the VFD for the information and instructions exchange and the overall machining activities are accessed to be monitored through the main supervisory computer equipped with Supervisory Control and Data Acquisition (SCADA) software. In addition to the locally supervision and control of the industrial process, there is access of controlling and monitoring of the system through IoT server via internet communication through the network interface provided to the main computer.

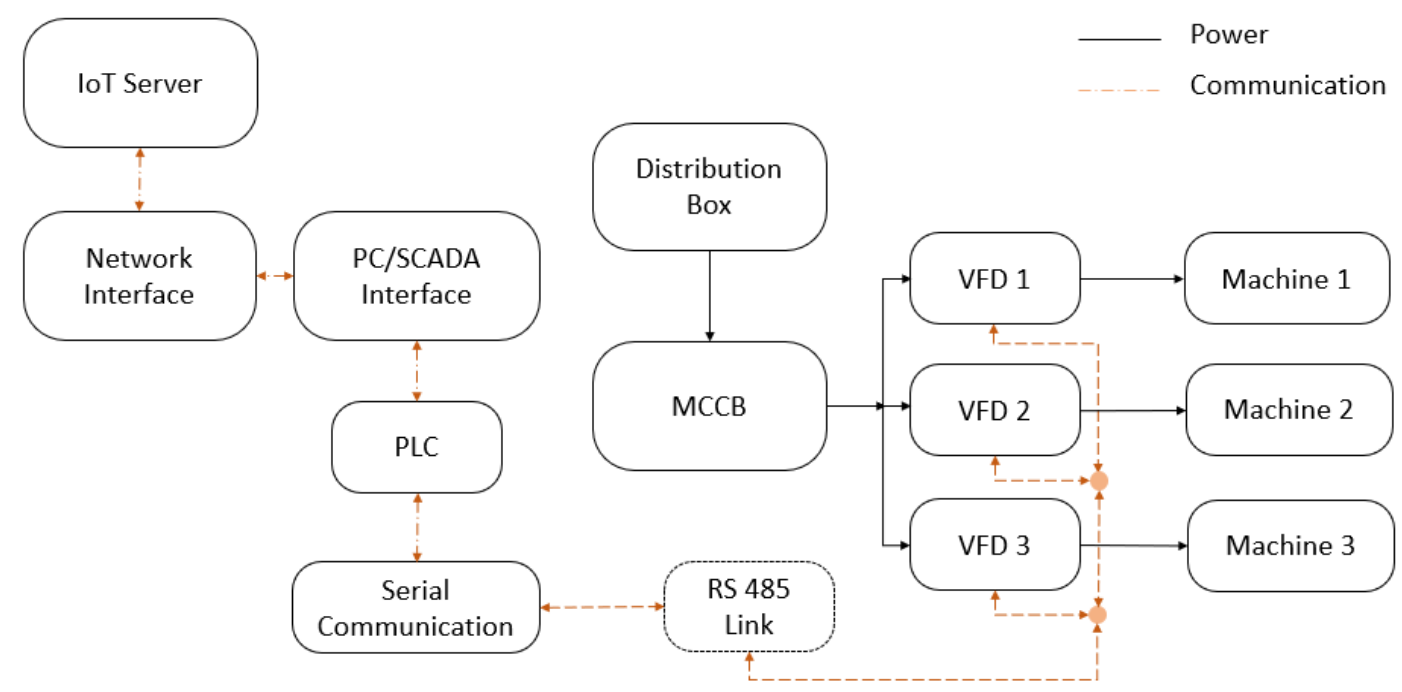

Fig. 1. Architecture of Proposed System 


\section{Experimental Implementation}

The experimental setup was carried out in the plant site of Himalayan Snacks and Noodles Pvt. Ltd. in Banepa, Nepal and the outcomes are recorded. The setup consisted of development of optimized control system by using the PLC for the logical decisions to be made to the system and was controlled from the supervisory control by the SCADA software developed in C\# environment in visual studio 2013. The end machining process were equipped with the infrared sensors to detect the objects in the rollers and to detect the machines status. The signals from the IR sensors were communicated with PLC for making necessary control commands based on the sensor status. The machine control is accessible from the push buttons located locally or from the SCADA interface from the main control computer. The control logic is made in XOR combination between SCADA system and the pushbuttons so that from any controller section the machine operations can be accessed easily.

\subsection{SCDA Software Design}

The C\# environment has been adapted in development of SCADA software to overcome the limitations of its use in small and medium scale industries due to the higher installation costs as discussed in chapter 5 before. The developed software has capability of communication to the IoT server through internet protocol and also communication to the master PLC through ethernet connection in local area network to monitor and control the system properties. The software is now developed to control and monitor three machining stations for the testing and validation purpose and can be increased as per the system requirements. The IoT server connected to this software allows the user to control the system from any internet connected device from any remote place and allows the data acquisition too.

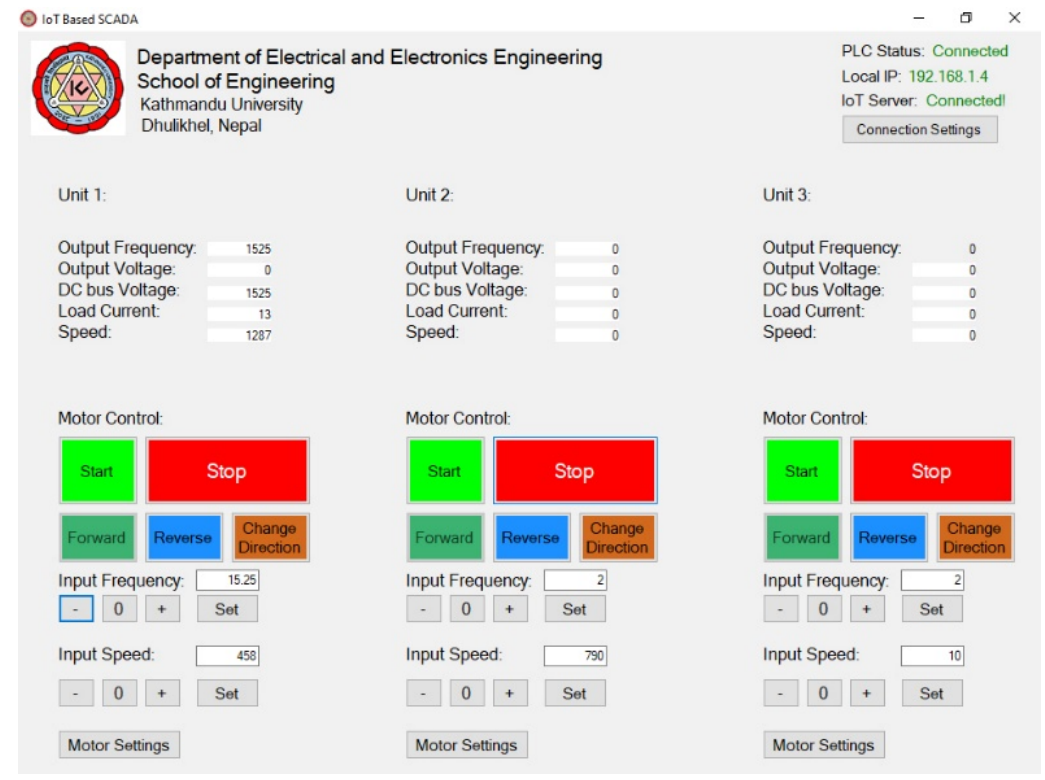

Fig. 2. User Interface of Main SCADA System 
In the Fig. 2, the layout of main screen user interface is shown. The status of PLC connection, status of IoT server connection, and local IP address to which the PLC is connected to SCADA system is displayed in the top right section of user interface. The three machining stations and their parameters are allowed to display in each row followed by their names. The set frequency, set speed is displayed in the input section as shown in figure. The monitored parameters like output frequency, output load current, DC bus voltage and the direction of rotation is also displayed there. The speed of the motor can be easily varied through setting the desired speed directly or setting the frequency in which the motor is desired to be running in the respective speed or frequency input section. The speed can be gradually increased or decreased by using increment or decrement button or reset to zero through the button ' 0 '. The increment/decrement rate per click can be set by motor settings button located at button of each motor control section in the main user interface. The 'motor setting' button pop-ups a new window allowing the users to set the basic parameters of the motor and save them to the application settings of the software so that in next start-up, these values are stored in the application settings of the software.

In the connection settings located at top right of the main interface, the user can set the IP address and the port of the connected PLC can also be set easily so that it can be setup in any customized system more quickly. By setting the appropriate IP address and port number in the settings window as shown in Fig. 3 (a), we can resolve the PLC connection error and the SCADA interface is now connected with the PLC through ethernet in local area network with specific IP address provided by the router. For this setup the IP address of the PLC is provided as "192.168.1.4" and port number " 502 " for data exchange between the main computer and the PLC. The basic parameters of the motors can be setup easily through the motor settings button and this pop-up a window as shown in Fig. 3 (b). The first starting speed of motor in rpm, maximum and minimum frequency of motor, frequency and speed change rate can be set in this window.

The errors occurred in the software are warned through the message box and can be resolved by addressing the respective errors easily. Fig. 4 shows the various stages of error dialogs appeared.
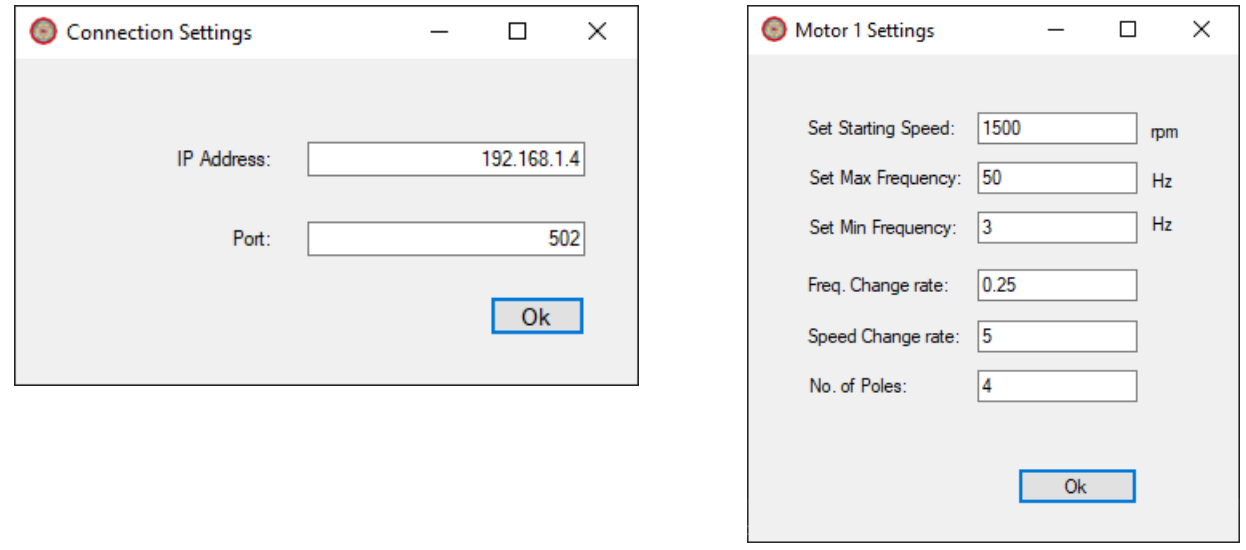

Fig. 3. (a) Connection Settings in main interface, (b) Motor Settings window

The system is made fully automatic and provided cloud-based control function to enable the flexible control and monitoring of the manufacturing system. The test setup for the given sections are provided with separate wiring as shown in figure Fig. 5. The power supply has been provided through 32A MCCB and is distributed in the circuit through the busbar. The MCB are installed for the overcurrent protection and short circuit protection in individual loads and the contactors are placed to turn the motors on and off from PLC logic or from the push buttons located locally near the motors which are combined in XOR logic so that any 
of the controller can make the necessary changes in the system. Also, PLC is allowed to communicate to the IoT cloud through the main computer connected through the SCADA interface to get and send the signals to the cloud so that it can be operated and monitored through the application of IoT.

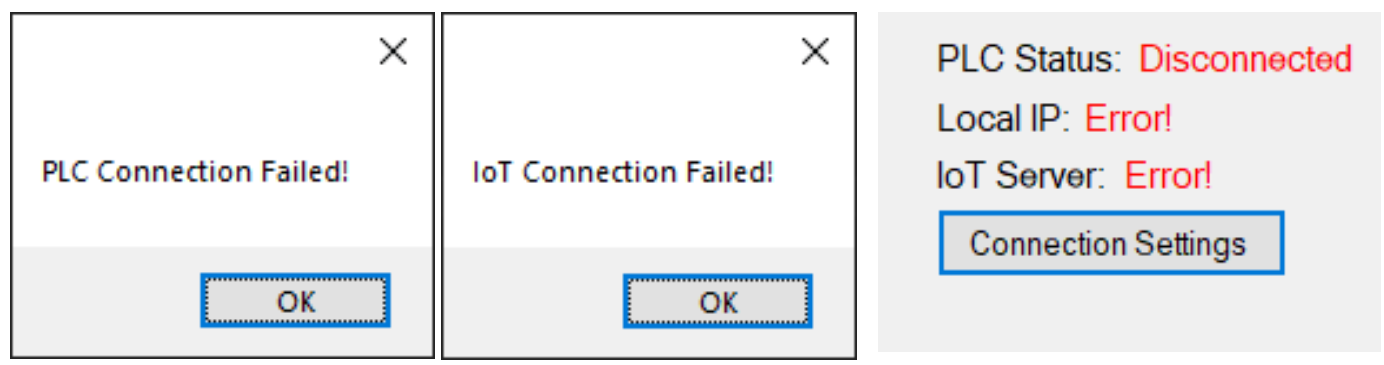

Fig. 4. Warning of PLC and IoT server connection Error

\subsection{Hardware Configuration}

The experimental setup and testing of the hardware were carried out in Himalayan Snacks \& Noodles Pvt. Ltd., Banepa plant site. The setup includes the wireless control and supervision of the roller, fryer and conveyor section of the plant. control and supervision of the roller, fryer and conveyor section of the plant. For the effective operation of the control system, the inputs from push buttons and the logical decisions from the PLC is programmed in XOR configuration so that any of the signal can set the desired operation of the system.

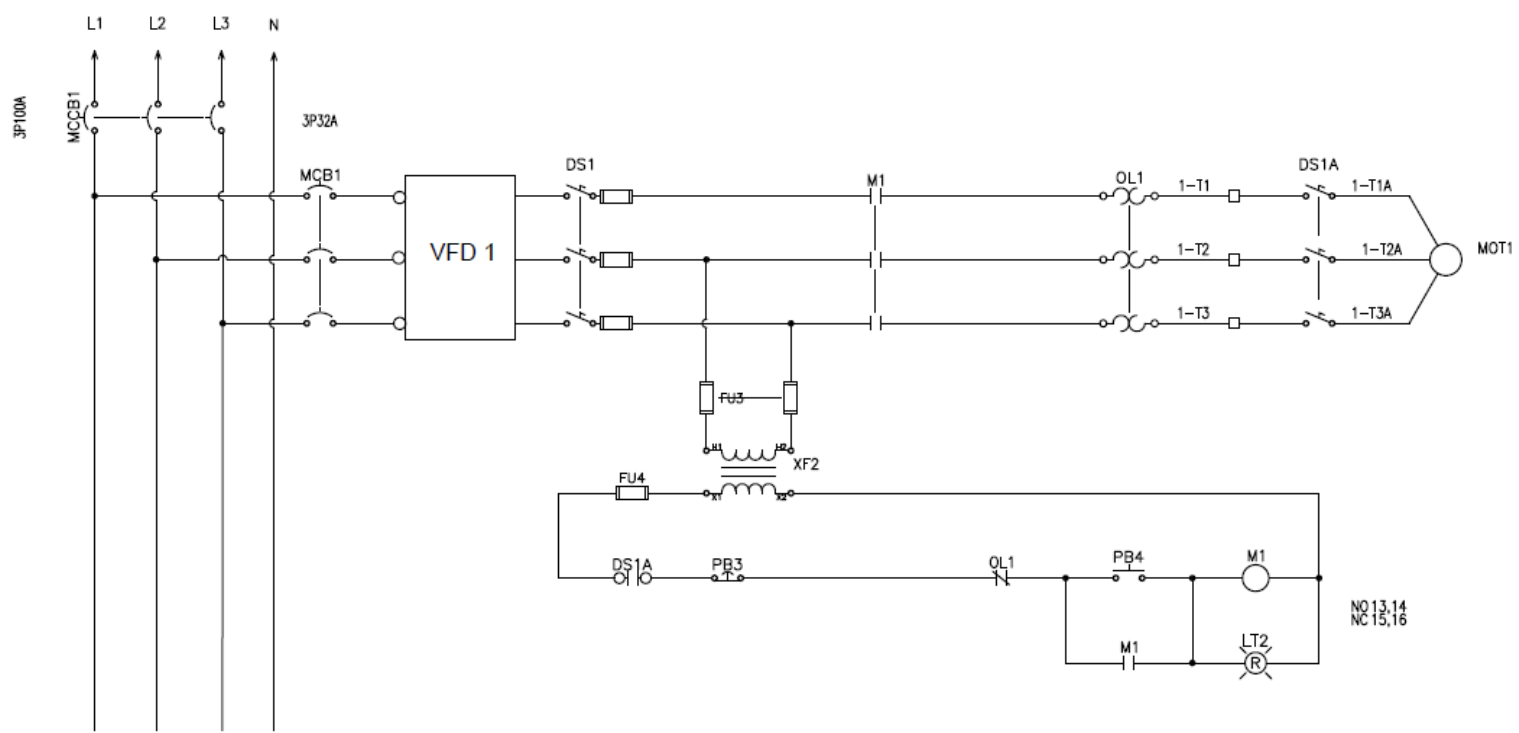

Fig. 5. Circuit Layout simplified for one Motor control system 
The Fig. 5 illustrates the circuit diagram of the test unit 1 and the same circuit was implemented for the rest 2 units. The system is made fully automatic and provided cloud-based control function to enable the flexible control and monitoring of the manufacturing system. The test setup for the given sections are provided with separate wiring as shown in Fig. 6 (a). The power supply has been provided through 32A MCCB and is distributed in the circuit through the busbar. The MCB are installed for the overcurrent protection and short circuit protection in individual loads and the contactors are placed to turn the motors on and off from PLC logic or from the push buttons located locally near the motors which are combined in XOR logic so that any of the controller can make the necessary changes in the system. Also, PLC is allowed to communicate to the IoT cloud through the main computer connected through the SCADA interface to get and send the signals to the cloud so that it can be operated. and monitored through the application of IoT.

The power is supplied to the VFDs in Fig. 6 (b) through the busbar in Fig. 6 (a) and the control function is given from the PLC through Modbus communication in parallel RS-485 communication with unique device id as 1,2,3 respectively. The particular machine can be instructed through the separate device ID and the read or write message command.

\subsection{Programmable Logic Controller Configuration}

For the proper communication between the field devices and the local and cloud-based controllers, and real time data and condition monitoring, PLC was installed to bridge the physical world with input and output units to the computing and data acquisition system.

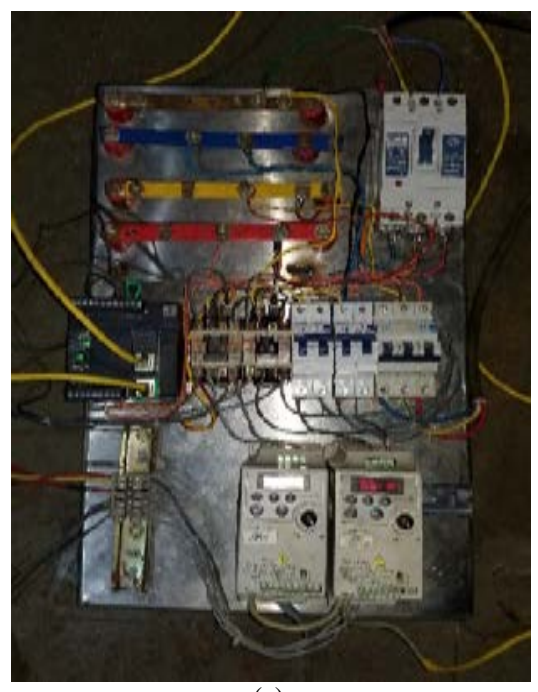

(a)

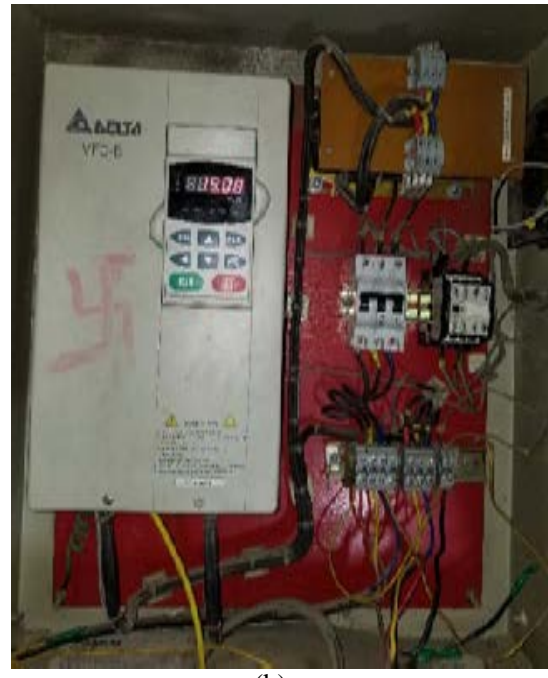

(b)

Fig. 6. Hardware configuration of developed system

The PLC was provided the inputs from the field sensors to get the real-time information of the machining units, the output pins were used to send the operation command to the actuators to perform the desired process. The serial Link is used to communicate and information exchange between the PLC and Variable Frequency Drives (VFD) to real-time data exchange of current running machines and their related parameters. All the information exchange through I/O pins, and serial link occurs automatically based on the ladder program defined in the PLC during the installation and it is allowed to be monitored, modified and override any 
instructions through the Supervisory Control unit, equipped with SCADA software in the master PC situated in the control room. This SCADA software is able to handle the control and data acquisition directly and also can be accessed through the internet of things application. This can operate in real-time form local unit and also from the cloud computing as well.

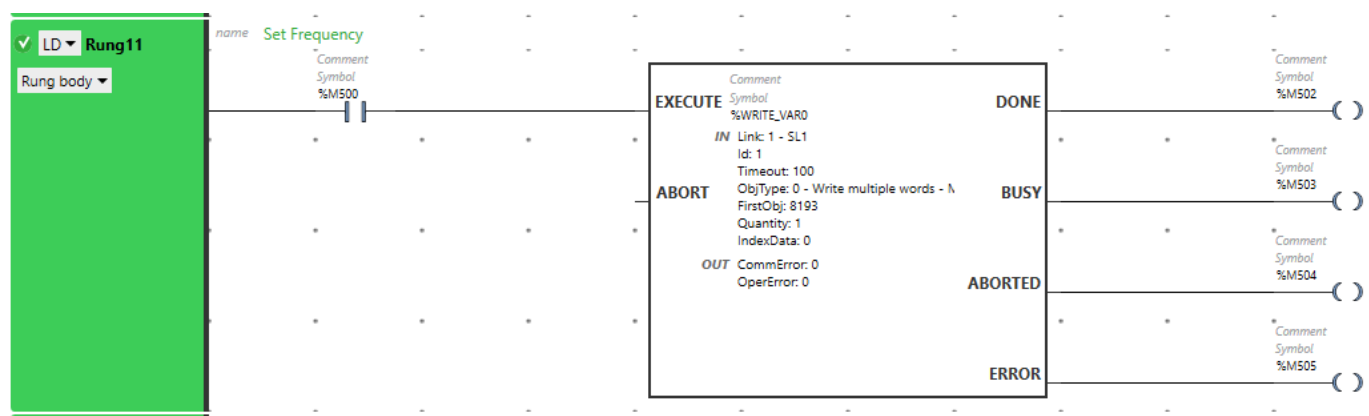

Fig. 7: Sample rung 11 of PLC ladder diagram to set desired frequency

\section{Data acquisition and system control}

For the data acquisition, visualization and control of the system through IoT devices the IoT server for this project has been temporally hosted in google firebase for testing purpose. The access to the IoT server is encrypted with the API key and passcode. With the pre-shared API key and passcode only the data in the server can be accessed and the necessary changes in the operation can be made. The IoT server can be hosted in own webserver for quick and more secured operation of the system.

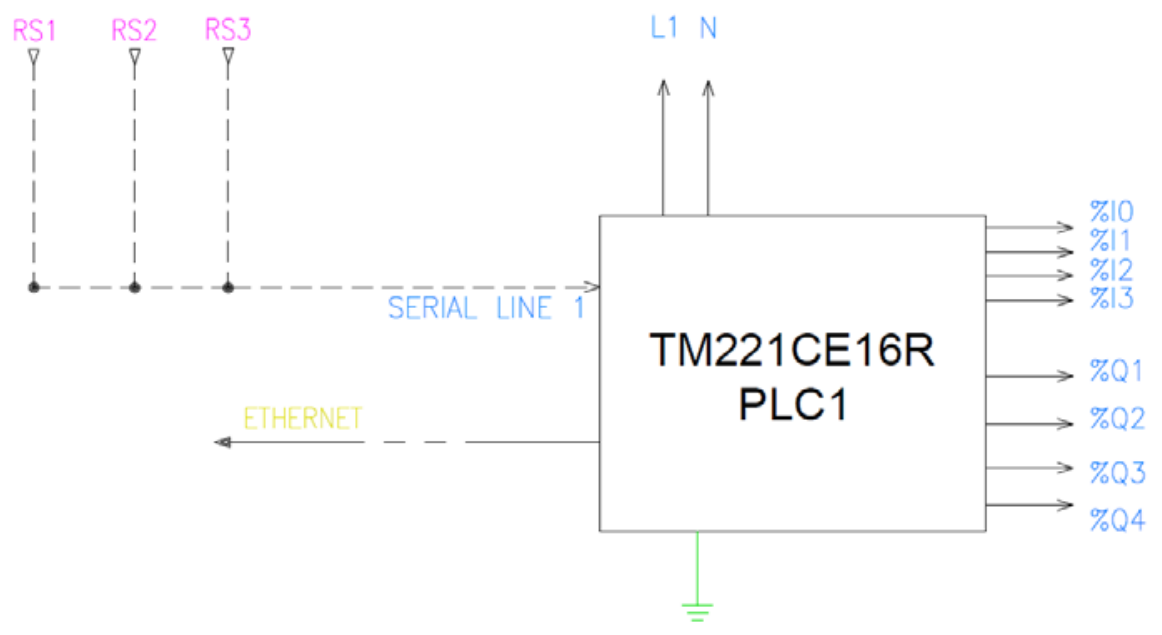

Fig. 8: PLC Wiring Diagram

For the remote operation of the system and operation monitoring, the android application has been also developed for easier visualization and control. The application can only access the function only after the successful validation of pre-shared API key and passcode. 
Table 1. Data Acquisition in SCADA System

\begin{tabular}{lllll}
\hline \multirow{2}{*}{$\begin{array}{l}\text { Frequency } \\
(\mathrm{Hz})\end{array}$} & \multicolumn{3}{c}{ Motor 1 } & \multicolumn{2}{c}{ Motor 2 } \\
\cline { 2 - 5 } & Current (A) & Voltage (V) & Current (A) & Voltage (V) \\
\hline 10 & 7.2 & 175 & 16.4 & 183 \\
15 & 15.3 & 192 & 7.1 & 202 \\
20 & 5.1 & 219 & 9.7 & 233 \\
25 & 5.4 & 236 & 10 & 255 \\
30 & 5.5 & 253 & 10.1 & 274 \\
35 & 5.5 & 270 & 8.7 & 295 \\
40 & 5.5 & 287 & 8.2 & 314 \\
45 & 5.4 & 307 & 7.1 & 333 \\
50 & 4.6 & 320 & 5 & 340 \\
\hline
\end{tabular}

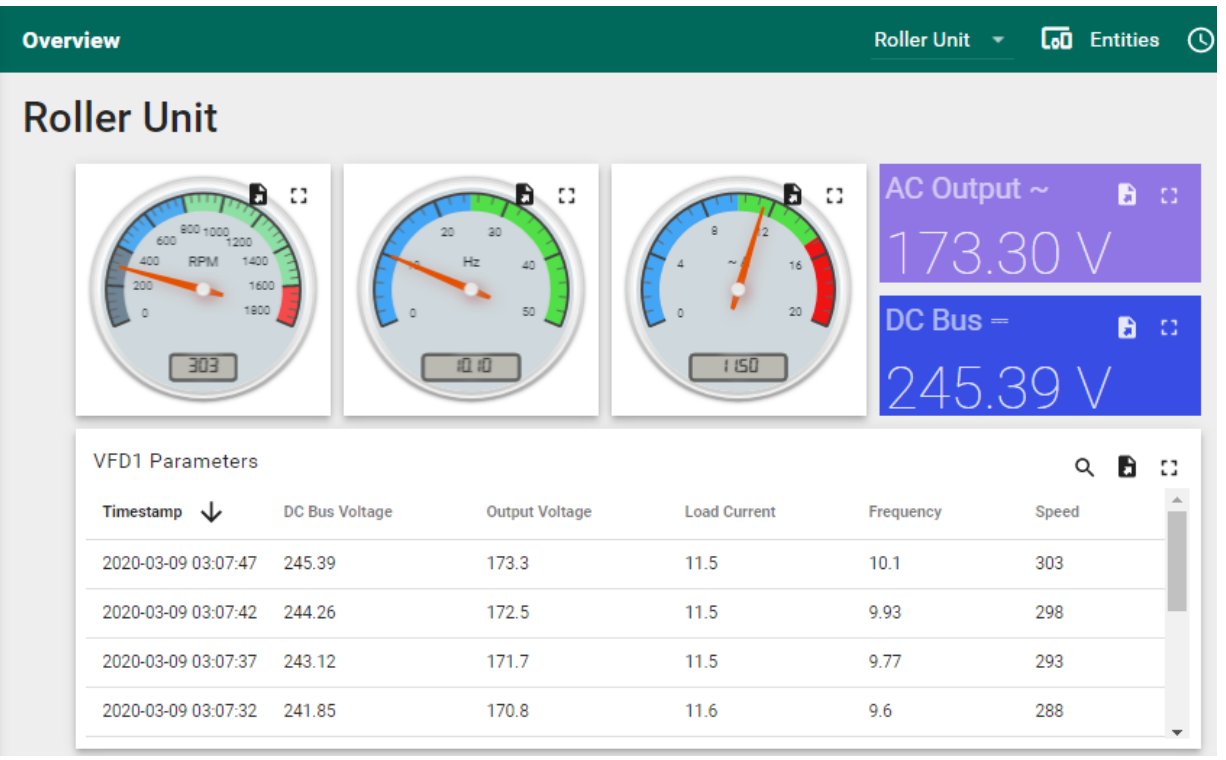

Fig. 9. Dashboard Showing real-time Data in IoT Interface

The data can be accessed and system control can also be done in mobile applications. The mobile application has feature of accessing the real-time data of the ongoing process, status of the running plant, access to change the control parameters of the machinery, change the operation and production plan of the industry and start and stop any machinery unit as per the requirement by the operator from the remote site.

\section{Conclusion and Discussion}

By using the platform of C\# environment and using Schneider M221 series PLC as RTU, we successfully tested the performance of low-cost SCADA system in in the milling and roller unit of Mayos Noodles plant in 
Banepa, Kavre. The setup was installed in the existing running unit of the industry by adding the infrared sensors to detect the flow of materials in the roller, ultrasonic sensors to detect the materials available in the mixer unit, PLC for logical controls and a SCADA interface to monitor and supervise the entire plant process. Additionally, the necessary electrical safety was provided by protection devices like MCCB, MCBs, overload relays, contactors and the add-in blocks, fuses etc. wherever required.

The setup includes the wireless control and supervision of the plant process through the use of internet and the plant process is made to be accessible to monitor and control through mobile applications and the web interface.

This can be easily implemented in small and medium scale industries, isolated and hybrid microgrid systems very easily with very low-cost investment compared to the commercial products.

All types of power generation, transmission and distribution system, industries and all types of process monitoring purpose, the emerging technology of supervisory control and data acquisition (SCADA) is being implemented and also the cloud-based control systems are also emerging in this field for the advanced monitoring and control of the process from the supervisory level. This reduces the workforce required, less time of action for decision taking, easy planning of system task, easy and proper visualization of the system process.

As the commercial SCADA products available in the market is expensive and higher in budget size for small and medium industries, we implemented a low-cost SCADA software developed in C\# environment and tested successfully in the process monitoring and control of Mayos noodles industry in Banepa. The test of this system has proved that we can reduce the cost investment in purchasing the software to monitor the industrial process and can run the industry in fully automatic and monitored environment. The product implemented has a huge possibility of upgrade and can be used in commercial industries to reduce their production costs by increasing the machines utilization, reduction in workforce requirement and can also improve the quality of products by monitoring the process more precisely.

The results obtained in this work can be implemented in the commercial and educational purpose to produce SCADA software and hardware for the monitoring and control of full industry. For more facilitated and expanded service, the SMS alerts can be sent to system administrators of any critical notifications, the user-interface can be developed more user friendly so that it can be operated from the webpage directly from using the selector switches and input data.

\section{Acknowledgements}

The authors would like to acknowledge Department of Electrical and Electronics Engineering, Kathmandu University for providing the required laboratory space and also like to thank Himalayan Snacks and Noodles Pvt. Ltd., Banepa for facilitating us for industrial setup and performance analysis of the developed product. Also, we would like to thank Mr. Ashish Shrestha, Mr. Ajay Singh, Mr. Tika Ram Regmi and Mr. Nishan Karki for their valuable contributions during testing and validation of the system as well as suggestions and review of the manuscript.

\section{References}

[1] L. O. Aghenta and M. T. Iqbal, "Low-Cost, Open Source IoT-Based SCADA System Design Using Thinger. IO and ESP32 Thing," Electronics, vol. 8, no. 8, p. 822, 2019.

[2] O. Carlsson, Engineering of IoT Automation Systems, PhD Thesis. Lulea, Sweeden: ESILAB, Lulea University of Technology, 2017. 
[3] S. Phuyal, J. Izykowski, D. Bista, and R. Bista, "Internet of Things in Power Industry: Current Scenario of Nepal," in International Symposium on Current Research in Hydropower Technologies (CRHT’IX), 2019, vol. 9.

[4] M. Hermann, T. Pentek, and B. Otto, "Design Principles for Industrie 4.0 Scenarios," in 49th Hawaii International Conference on System Sciences (HICSS), Koloa, HI, USA, 5-8 Jan. 2016.

[5] "Factories of the future, Multi annual roadmap for the contractual PPP under horizon 2020," European Commission, Luxembourg: Publications Office of the European Union2013.

[6] "European Roadmap for Industrial Process Automation," European Regional Development Fund [available: www.processit.eu/roadmap]Sept. 2018.

[7] A. Mercurio, A. Di Giorgio, and P. Cioci, "Open-source implementation of monitoring and controlling services for EMS/SCADA systems by means of web services-IEC 61850 and IEC 61970 standards," IEEE Transactions on Power Delivery, vol. 24, no. 3, pp. 1148-1153, 2009.

[8] A. M. Alihussein, "A Supervisory Control And Data Acquisition (SCADA) for Water Distribution System of Gaza City," M. Sc. Thesis, The Islamic University of Gaza, 2010.

[9] H. A. A. Ahmed and A. Ahmed, "Efficient Web-Based SCADA System," Automation Engineer in Qena Paper Company, 2011.

[10] O. Carlsson, "Enabling Large IoT Platforms in Industrial Process Automation," Luleå tekniska universitet, 2015.

[11] R. I. Rajkumar, T. J. Alexander, and P. Devi, "ZigBee based design of low cost SCADA system for industrial process applications," in 2016 IEEE International Conference on Computational Intelligence and Computing Research (ICCIC), 2016, pp. 1-4: IEEE.

[12] T. Mononen and J. Mattila, "A low-cost cloud-extended sensor network for supervisory control," in 2017 IEEE International Conference on Cybernetics and Intelligent Systems (CIS) and IEEE Conference on Robotics, Automation and Mechatronics (RAM), 2017, pp. 502-507: IEEE.

[13] M. Avhad, V. Divekar, H. Golatkar, and S. Joshi, "Microcontroller based automation system using industry standard SCADA," in 2013 Annual IEEE India Conference (INDICON), 2013, pp. 1-6: IEEE.

[14] A. S. Prokhorov, M. A. Chudinov, and S. E. Bondarev, "Control systems software implementation using open source SCADA-system OpenSCADA," in 2018 IEEE Conference of Russian Young Researchers in Electrical and Electronic Engineering (EIConRus), pp. 220-222: IEEE.

[15] D. F. Merchán, J. A. Peralta, A. Vazquez-Rodas, L. I. Minchala, and D. Astudillo-Salinas, "Open source SCADA system for advanced monitoring of industrial processes," in 2017 International Conference on Information Systems and Computer Science (INCISCOS), 2017, pp. 160-165: IEEE.

[16] L. O. Aghenta and M. T. Iqbal, "Development of an IoT Based Open Source SCADA System for PV System Monitoring," in 2019 IEEE Canadian Conference of Electrical and Computer Engineering (CCECE), 2019, pp. 1-4: IEEE. 


\section{Authors’ Profiles}

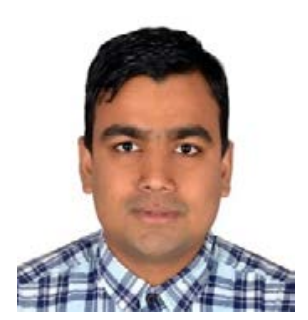

Sudip Phuyal completed his bachelor in Electrical and Electronics Engineering from Pokhara University, Nepal in 2016 and currently enrolled in MS by Research candidate at Department of Electrical and Electronics Engineering, Kathmandu University and also working as project member and technical expert in the European Union and Royal Norwegian Embassy at Nepal funded research project "Micro-Grid Management and Control Systems for the Himalayan Region of Nepal”. He is also an Erasmus+ scholar for research exchange to Wroclaw University of Science and Technology, Poland under the Erasmus Mundus grants funded by European Union in 2018. He has also been awarded with the research grant funded by Handong Global University, South Korea for the development of low speed PM generators to install in the traditional water mill of rural village of Nepal in 2016. He is also a founder of Robotics Academy of Nepal (RAN), former R\&D advisor of Innovative Ghar Nepal, and also, currently working as senior electrical and instrumentation engineer at Himalayan Snacks and Noodles Pvt. Ltd. with major responsibilities of design and supervision of electrical power system and process control of the industry. His research interest includes control system, process automation, industrial robotics, power electronics, electric drives and traction.

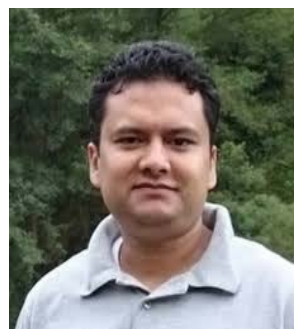

Diwakar Bista received his Bachelor degree in Electrical and Electronics Engineering (Specialization in Power and Control), and MS by Research degree from School of Engineering, Kathmandu University. Currently, he is pursuing his $\mathrm{PhD}$ from the same institution, and working as an Assistant Professor at Department of Electrical and Electronics Engineering. His research interests include the Lighting Technologies, Control and Instrumentation, Renewable Energy etc. In the past, he had coordinated number of projects funded by Royal Norwegian Embassy at Nepal, European Union, Nepal Government. He is currently the Project Leader of the Center for Electric Power Engineering (CEPE), Kathmandu University. He is also working as a Coordinator from Nepal of problem-based learning project funded by Erasmus+ program of EU.

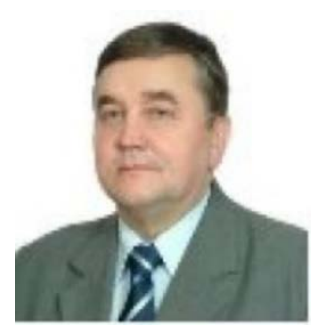

Jan Izykowski received his M.Sc., Ph.D. and D.Sc. degrees in Electrical Eng. from the Faculty of Electrical Engineering of Wroclaw University of Science and Technology (WUST) in 1973, 1976 and in 2001, respectively. In 1973 he joined Institute of Electrical Engineering of the WUST. Presently he is a Professor and Director of this Institute. His research interests are in power system simulation, power system protection and control, and fault location. 


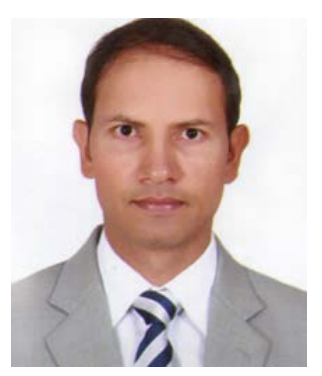

Rabindra Bista received his B.Sc. IT from Tribhuwan University, Nepal in 2004, M.E and Ph.D. in Computer Engineering in 2007 and 2011 respectively from Chonbuk National University, South Korea. He is an Assistant Professor at Department of Computer Science and Engineering, Kathmandu University since 2011. His research interests include Wireless Sensor Network, IoT and IPv6, Health Informatics, Telemedicine, e-Health, m-Health and Natural Language Processing.

How to cite this paper: Sudip Phuyal, Diwakar Bista, Jan Izykowski, Rabindra Bista. "Design and Implementation of Cost Efficient SCADA System for Industrial Automation", International Journal of Engineering and Manufacturing(IJEM), Vol.10, No.2, pp.15-28, 2020. DOI: 10.5815/ijem.2020.02.02. 